\title{
Enhanced Reputation-based Tit-for-Tat Strategy for Collaborative Social Applications
}

\author{
Ahmed Al-Dhanhani*, Hadi Otrok ${ }^{\dagger}$, Rabeb Mizouni ${ }^{\dagger}$, Ahmad Al-Rubaie* \\ *Etisalat BT Innovation Centre, Abu Dhabi, UAE \\ ${ }^{\dagger}$ Khalifa University of Science, Technology and Research, Abu Dhabi, UAE
}

\begin{abstract}
Social applications have witnessed a rapid growth in their use. Millions of people are utilizing them on a daily basis in order to share their experience, information and to communicate with their family members and friends. Lately, these technologies have been used to foster collaboration in education, however, it is a case of hit and miss and without established techniques to ensure or replicate success. One known problem that can impact the sustainability of collaborative social applications is the presence of selfish users. A selfish user adopts a free riding behaviour that takes advantage of the collaborative group without contributing back which would affect the group's survivability. However, the inability to contribute back is not necessarily due to selfishness. In fact, cooperative learners may avoid participating due to the lack of expertise and/or confidence. In the literature, repeated non-cooperative game theory was introduced as a solution where several strategies were introduced to identify selfish users. However, such strategies do not differentiate between selfish learners and cooperative learners who are reluctant to answer others' requests either due to lack of knowledge or confidence. In this paper, we show that educational and collaborative groups need to distinguish between those types of users and put in place mechanisms to encourage non contributing cooperative learners to participate rather than punishing them and excluding them from the group. An enhanced reputation-based Tit-for-Tat strategy is proposed as a solution that will enhance the group activity and overall gain.
\end{abstract}

Index Terms-Game Theory, Social Applications, Tit-for-Tat, Free Riding, Collaborative Learning, Collaborative Groups.

\section{INTRODUCTION}

Over the past few year, social applications have gained significant popularity on the Internet and a great deal of user generated content is now available within these applications and being accessed and consumed by a plethora of users globally. Many have identified opportunities that leverage the popularity of social applications in other fields, such as education; and educators have already started working on using social applications to foster collaboration among groups of learners. These groups can exist in forums, emails, chat systems, questions and answers sites, and most recently social networking sites.

Currently, the success of social applications for collaboration has not been consistent and the ability of these social applications to stimulate their subscribers to contribute varies greatly. Encouraging participants to contribute has been more of an art than science [1]. There is a combination of factors that have been studied and claimed to result on guaranteeing the active contribution of the participants ranging from psychological to economical views [1]. It is not an easy task to create a controlled study to test one factor in separation from the others in a real online community. In this paper, we adopt the economical view by assuming that users are rational agents aiming at maximizing their benefits which increase when other users collaborate with them.

Achieving collaboration is, in fact, a key success factor for educational social applications. Unfortunately, over the time learners participation may diminish or even stop because they may lose interest on the theme of the discussion and/or they do not get satisfactory answers to their requests. Selfish behaviour could also arise where some participants will try to take advantage of the group and request help from the other participants while ignoring answering requests of others to maximize their benefit. Such situation is referred to as the tragedy of the commons [2]. Over the time, the existence of such free riding behaviour may demotivate participants from collaborating and may affect the survivability of the group caused from a social dilemma [3].

One solution would be to distinguish between the cooperative participants and the free riders, and motivate the participants to collaborate with the cooperative users while punishing the free riders by not cooperating with them [4]. However, in an educational social application, it is not always clear if a user is free riding or lacking the knowledge to answer some requests. Sometimes, a cooperative user might be punished as a result of this. Therefore, there is a need to develop a higher level of granularity to distinguish between cooperative users who lack the knowledge and non cooperative users to avoid punishing those who are cooperative and end up demotivating them.

As a solution, game theory is used to formulate the interaction among all participants as a repeated non-cooperative game based on Tit-for-Tat strategy. In our investigation, we create a model where a number of participants formed a group inside a social application for the purpose of collaboration. We solve the problem of the free riding behaviour by enticing the cooperative users to follow a strategy that maximizes their gain and limits the gains of the free riders.

Reputation has been used a lot in different web applications to give a good indication of whether a user is cooperative or not. Therefore, we model our problem using the reputation- 
based Tit for Tat strategy to distinguish between cooperative users from the free riders. Our analysis shows that having the reputation might not be enough to distinguish all cooperative users as it might result in injustice to a number of cooperative users by considering them as free riders.

To address this problem, we introduce a points system that compliments the reputation system where the user is given points for the contributions. Such points can help users to identify themselves as not selfish and respond to requests that cannot be answered by them through getting the answer from others. This gives the user a second chance to prove that $\mathrm{s} / \mathrm{he}$ is cooperative even when s/he lacks the knowledge to answer a request. This will prove to the system that the user is still cooperative so that the system will stabilize the reputation. The results of our investigation prove that this addition will improve the gains of cooperative users and help differentiate them from free riders.

In summary, the main contribution, of our paper, is a a game theoretical model that is a able to:

- Simulate a collaborative group using the reputation based Tit for Tat strategy to decrease the free riding behaviour, and

- Develop a higher level of granularity to distinguish between cooperative users who lack the knowledge and non cooperative users

The rest of the paper is structured as follows. Section II presents the game theory model. In section III, we show the the reputation based Tit for Tat strategy, then we add the points system to it and analyze the results. Section IV presents some related work in the field of game theory and social applications. Finally section V concludes the paper.

\section{GAME Definition}

In a social application, users can cooperate by helping others in their requests, by participating in their discussions or simply commenting in their contributions. Still, users can also defect from cooperating and not contribute. We propose to model the users as a game to study their behaviour when using a reputation based Tit for Tat strategy.

Using game theory, this situation of cooperating and defecting can be modeled as a non-zero non-cooperative game [5]. It's modeled as a non-zero because the benefit can be shared rather than being strictly transferred to one person only. Also, it was modeled as a non-cooperative because each person makes his decision independently from the other.

TABLE I

PRISONER'S DILEMMA PAYOFF MATRIX

\begin{tabular}{|c|c|r|}
\hline & Cooperate & Defect \\
\hline Cooperate & $\mathrm{R}(-1), \mathrm{R}(-1)$ & $\mathrm{S}(-10), \mathrm{T}(0)$ \\
\hline Defect & $\mathrm{T}(0), \mathrm{S}(-10)$ & $\mathrm{P}(-5), \mathrm{P}(-5)$ \\
\hline
\end{tabular}

Such games are usually modeled against the famous Prisoner's Dilemma game invented by two Rand corporation scientists in 1950 [6]. The prisoner's dilemma game represents the situation of two criminals caught by the police at the same time. These criminals have two strategies to independently select from. They can either confess (defect) or not (cooperate). The results of the possible outcomes is outlasted in table I where:

1) R: Reward is to be prisoned for 1 year

2) P: Punishment is to be prisoned for 5 years

3) S: Sucker is to be prisoned for 10 years

4) $T$ : Temptation is to be set free.

These constants must satisfy the following two conditions:

1) $T>R>P>S$

2) $2 R>T+S$.

Using the dominant strategy technique, the best strategy for a criminal when both criminals do not know the other's decision is to "defect" to avoid the sucker which is the worst case since $T>R>P>S$. However, in the case of playing this game for infinite number of times, the best strategy will be to "cooperate" since $2 R>T+S$.

TABLE II

ASYNCHRONOUS GAME MODEL

\begin{tabular}{ll}
\hline Payoffs & \\
\hline Reward & $R=a+b$ \\
Punishment & $P=c+d$ \\
Temptation & $T=c+b$ \\
Sucker & $S=a+d$ \\
\hline Considering & \\
\hline$a$ & The cost of cooperating in a turn \\
$b$ & The reward from being cooperated with in \\
& a turn \\
$c$ & The cost of defecting in a turn \\
$d$ & The cost of not being cooperated with \\
\hline
\end{tabular}

The prisoner's dilemma game as is cannot resemble a collaborative group where decisions between two participants are not simultaneous but taken in two different stages. A modified version of the prisoner's dilemma game which is the iterated asynchronous prisoner's dilemma game has been introduced in the literature. To accommodate these changes in the game, we can use the model proposed by Nowak[?] summarized in table II.

These values should be calculated so that they do not violate the previously mentioned criteria of the prisoner's dilemma.

\section{A. Collaborative Game Settings}

In this section we explain the settings and introduce the assumptions we considered when formulating the game. Consider that we have a group of $(N)$ participants in a social application, each user is a member of $m$ groups and posts made by users are restricted to groups. Each user is able to:

1) Post a request.

2) Answer others' requests.

3) Ignore a request because s/he is not able to answer it.

4) Ignore a request although s/he is able to answer it. Such a behaviour is known as "selfish" or "free riding". 
We also assume that :

1) The social application is keeping track of all requests and responses of each participant.

2) The social application is updating each user with the cooperation status of the other users.

3) The group lifetime is infinite.

4) All participants are active.

5) The community is well established and active and in a state where it is in need of more answers rather than requests.

6) Any request may have more than one answer.

Our game will run for 10000 iterations. At each iteration $(t), \frac{1}{10}$ of the users in the group will be chosen randomly to have requests $Q_{i q}(t)$. The rest of the users can either respond to such requests or ignore them either because they want to free ride or because they are not able to answer. Our model considers ignoring requests as a defection regardless of the reason.

In total, we have $(N)$ participants where $\left(N_{c}\right)$ of them are cooperative and $\left(N_{s}\right)$ are selfish where $N=N_{c}+N_{s}$. For each interaction between two participants, a non-requesting cooperative participant $j$ would offer one useful response with a probability of $P_{a}$ considering s/he had the time to see the question under the probability $P_{s}$. Each participant has his/her own $P_{a}$ value ranging from 0 to $\frac{1}{4}\left(0<P_{a}<\frac{1}{4}\right)$ and also his/her own probability $P_{s}$ ranging from 0 to $\frac{1}{2}\left(0<P_{s}<\frac{1}{2}\right)$. The values of $P_{a}$ and $P_{s}$ are distributed normally between the participants to simulate the difference between them. The variability of $P_{a}$ simulates the probability of a participant ability to answer a request because he may not have enough knowledge, and $P_{s}$ simulates the probability of a participant ability to answer a request because he may not have time to answer it or he may not have the chance to see the request. III.

A summary of these game elements is described in Table

In the following, we define our game parameters that can fulfil the conditions of the asynchronous repeated prisoners dilemma game stated in table II:

1) Gain from getting a response, $b=10$

2) Cost of responding to a request, $a=-1$

3) Loss from not getting any response to the request, $d=$ $-10$

4) Cost of ignoring a request, $\mathrm{c}=0$

Note that the parameter $a$ is given a negative value to represent the cost of responding to a request since it requires effort and time to write an answer. According to our settings, $c$ is greater than $a$. It reflects the fact that ignoring a request gets a higher benefit at one time. Furthermore, $c-a$ is less than $b-d$ showing that the cost of responding is less than the benefit of getting a response. Therefore, for the longer term, rational users are better off cooperating with each other. These parameters ensure that the results would give an indication on the survivability and the efficiency of the group for all cooperative users. Also, it is worth to note that these parameters show the calculations made per request.

\section{REPUTATION-BASED TIT FOR TAT WITH POINTS SYSTEM}

In this section, we will present simulations of a collaborative group who adapted the reputation based Tit for Tat strategy. Then we will introduce the points system and assess the benefits of this addition to the reputation based Tit for Tat strategy.

\section{A. Reputation based Tit for Tat}

With the flows of the classical and generous Tit for Tat strategies in collaborative groups[7] and the expanding usage of reputation in different applications, we analyze a number of users who adopted the reputation based Tit for Tat strategy inside a collaborative group.

Considering the reputation equation, many equations have been used differing according to the domain and requirements of the community. We only use the simple way of dividing the number of times the user has answered a request over the number of times the user has requested to differentiate between the cooperative users and the free riders. The focus here is not to produce the equation, but is more about enticing the strategy of using a reputation based Tit for Tat approach. Therefore, a system designer should come up with the equation that suits the community best depending on its domain and requirements.

In game theoretical terms, using this strategy a player $j$ in his decision $D_{j i}(t)$ to cooperate with player $i$ or not; he will take in consideration the total number of times he had requests till time $t, N Q_{j}(t)$ and also the number of times player $i$ decided to cooperate with player $j$ and responded to his requests till time $t, N D_{i j}(t)$. Using these values we will have the ratio in equation 1

$$
\frac{N D_{i j}(t)}{N Q_{j}(t)}
$$

For player $i$ to be considered cooperative so that player $j$ would cooperate with him, this ratio should be above a certain value. This value will be dependent on a constant $\delta$ that we will choose its value. Also, all of this mainly depends on player $j$ having the answer $A_{j, i}(t)$ to the request of player $i$ at time $t$ and to have seen the request of $i$ at time $t, S_{j i}(t)$. This can be resembled in equation 2 .

$$
D_{j i}(t)=A_{j i}(t) \quad . \quad S_{j i}(t) \quad \text { if } \quad \frac{N D_{i j}(t)}{N Q_{j}(t)}>\delta
$$

Depending on the value we choose for $\delta$, the selfish users in order to imitate the cooperative behaviour will have to increase their cooperation to almost the level of the other cooperative users. We also give an initialisation phase of 500 iterations for the system to have a chance to calculate initial reputation values between the users.

Fig. 1 shows the total system gain in the case of using the reputation Tit for Tat strategy with 100 users with the lines 
TABLE III

SUMmARY OF THE GAME ELEMENTS

\begin{tabular}{ll}
\hline Game Model Elements & \\
\hline Players & Participants of a group. They can be selfish(free riders) or cooperative. \\
Cooperate & Responding to other participants requests(posts) \\
Defect & Ignoring/not answering other participants requests \\
Selfish User & Posts new questions to others but does not answer others' requests \\
$N_{s}$ & Number of selfish users \\
Cooperative User & Requests from others and answers others' requests \\
$N_{c}$ & Number of cooperative users \\
$P_{r}$ & Probability of a user requesting in a single turn which equals $\frac{1}{10}$ \\
$P_{a}$ & $\begin{array}{l}\text { The probability of a player to know the answer of a request and ranges } \\
\text { from } 0 \text { to } \frac{1}{4}\end{array}$ \\
$P_{s}$ & $\begin{array}{l}\text { The probability of a player to have the time to see a request and ranges } \\
\text { from } 0 \text { to } \frac{1}{2}\end{array}$
\end{tabular}

Number of iterations $i \quad$ Number of times the process of requesting is repeated

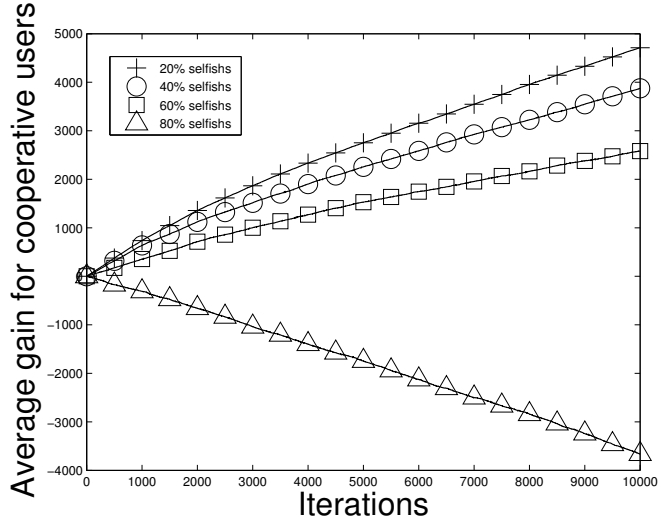

Fig. 1. Comparison between the different percentages of selfish users in reputation based Tit for Tat with $\delta=0.01$ in the average gain of cooperative users with an initialisation phase for 500 iterations

representing $20 \%, 40 \%, 60 \%$ and $80 \%$ of selfish users and $\delta$ $=0.01$.

We can see that higher percentages of selfish users lead to lower average gain for the cooperative users. This is expected since the higher the number of cooperative users, larger the chance of getting gain from cooperating with each other.

We can conclude that if learners adopt a reputation Tit for Tat like strategy, the collaboration would increase. It even limits the benefits that free riders could get in the case of the generous Tit for Tat strategy[7]. However, there is still

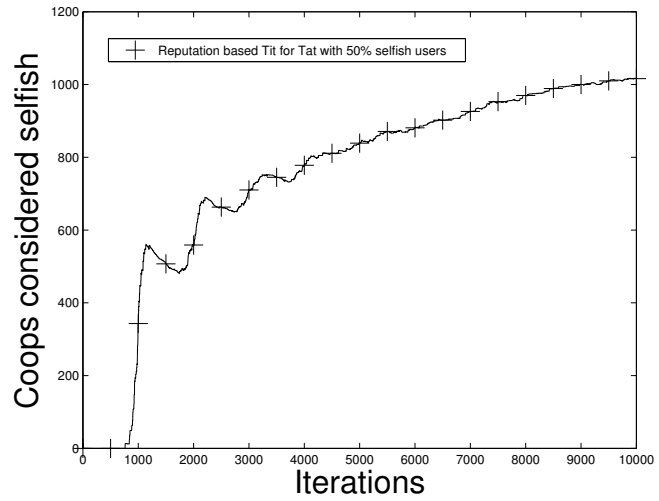

Fig. 2. Number of times a cooperative user is considered selfish by another cooperative user with $50 \%$ selfish users, $\delta=0.01$ and an initialisation phase for 500 iterations

a large enough probability that a cooperative user will be considered selfish by another cooperative user as seen in fig. 2 . There is a need for these cooperative users to have another chance to prove that they did not defect because of a free riding behaviour.

\section{B. Introducing the points system}

The idea behind the points system is to give the cooperative users a second chance to prove that they are not free riding. The user is given points by the system for his contributions. In the situations where a user sees a request but is not able to answer it because s/he does not have the knowledge to do 
so, s/he will have the option to pay points to the system to trigger an action from the system side to help this request get answered. This action will only stabilise the reputation of the user so it does not drop down.

We add an assumption that a cooperative user will not use the points unless s/he is getting near the point of being considered selfish. In more details, the user will check if s/he defected for the next $k$ number of requests from the other user, will s/he still be considered cooperative or not. If the user expects that $\mathrm{s} / \mathrm{he}$ can be considered selfish after $k$ number of requests, the user will pay points to the system to keep the reputation stable on the condition that $\mathrm{s} / \mathrm{he}$ has points available to use, $P_{\text {available. }}$. The number of points a user receives from contributing is only one point, $P_{\text {award }}=1$, and the number of points the user pays to stabilise his/her reputation is also one point, $P_{\text {consume }}=1$. This is resembled in equation 3 .

$$
D_{j, i}(t)=\left\{\begin{array}{ccrll}
\text { if } & A_{j, i}(t) & S_{j, i}(t) & \text { if } & \frac{N D_{i j}(t)}{N Q_{j}(t)}>\delta \\
& \text { else } & S_{j, i}(t) & \text { if } & P_{\text {available }}>0
\end{array}\right.
$$

If the user used the points, his/her number of points will reduce by $P_{\text {consume }}$, which will result in $P_{\text {available }}=$ $P_{\text {available }}-P_{\text {consume }}$.

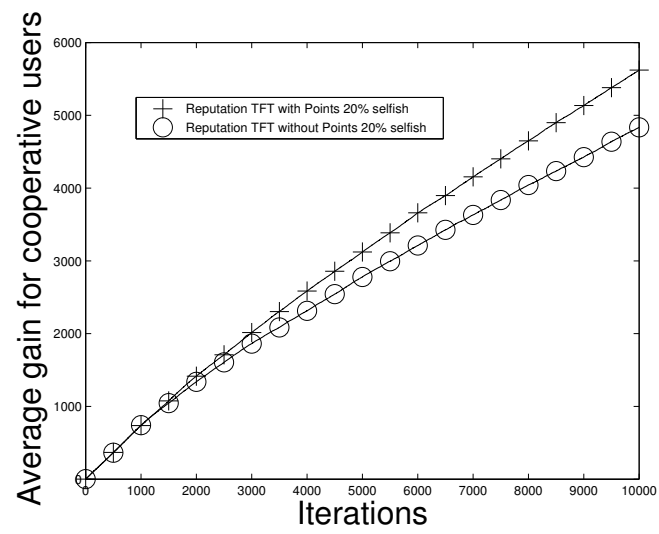

Fig. 3. Comparison between Reputation Tit for Tat with the use of points and without the use of points with $20 \%$ selfish users in the average gain of cooperative users, $\delta=0.01, k=5$ and an initialisation phase for 500 iterations

Fig. 3 shows the affect of adding the points system to the reputation based Tit for Tat strategy. The gap between the two lines is the gain that the cooperative users are able to get from introducing the points system in the case of having $20 \%$ selfish users only.

With larger number of selfish users, the gap becomes even larger, hence, the points system is even more important as seen in fig.4. This shows that it becomes harder to distinguish between cooperative and selfish users when increasing the numbers of selfish users. Hence, giving another chance to the cooperative users becomes even more important.

The reason behind the increasing gain in using points is that it causes less numbers of cooperative users to be considered

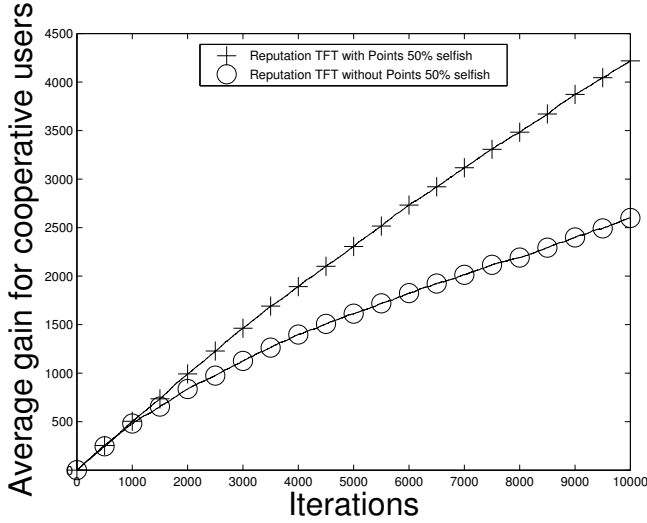

Fig. 4. Comparison between Reputation Tit for Tat with the use of points and without the use of points with $50 \%$ selfish users in the average gain of cooperative users, $\delta=0.01, k=5$ and an initialisation phase for 500 iterations

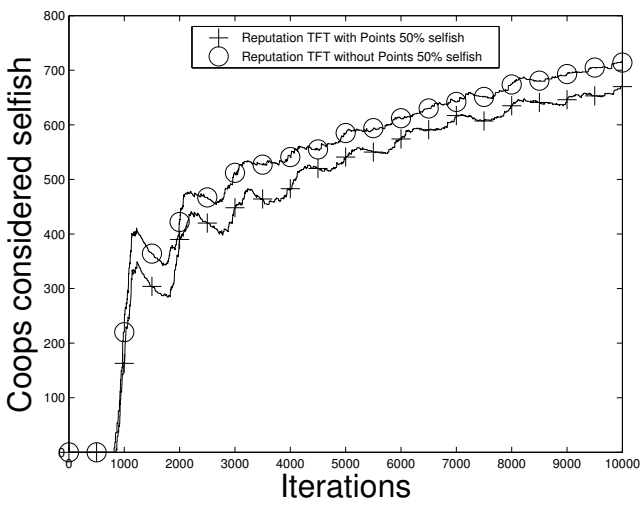

Fig. 5. Comparison between using points and not using points in the number of times a cooperative user is considered selfish by another cooperative user with $50 \%$ selfish users, $\delta=0.01, k=5$ and an initialisation phase for 500 iterations

selfish by other cooperative users as in fig.5. Moreover, we can reduce this number more by awarding the cooperative users more points. In the previous simulations, $P_{\text {award }}$ was always equal to one, but increasing it can yield different results.

Fig.6 shows a comparison between different values of $P_{\text {award }}$, it is clear that by giving the cooperative users more points, they have more chances to prove that they are cooperative later on. The problem with this approach is, by giving too many points, free riders may try to get use of it similarly to how they are able to get use of the generosity of the users in the generous Tit for Tat strategy. Also, large number of points in the system could cause an inflation, making the value of the points decrease with time. This is a common problem in systems with virtual currencies that are injected from outside the system[1]. Therefore, the system designer must adjust the value of the awarded points $P_{\text {award }}$ to the degree where it is optimised for the community. Also, if it happens that the value of the points decreased, the system designer can raise the cost 


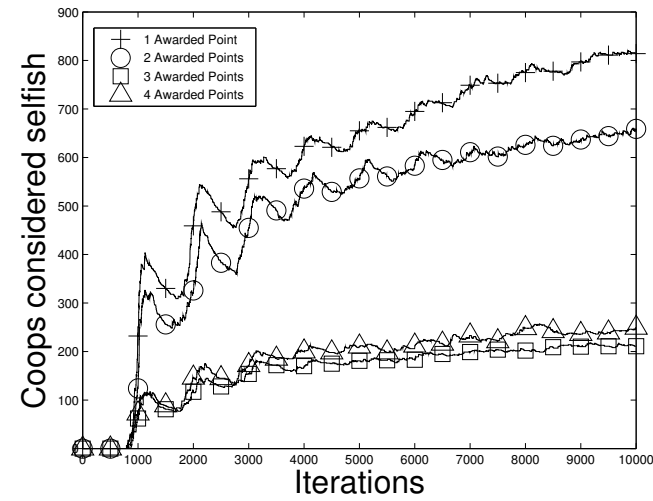

Fig. 6. Comparison between using different numbers of awarded points when using points in the number of times a cooperative user is considered selfish by another cooperative user with $50 \%$ selfish users, $\delta=0.01, k=5$ and an initialisation phase for 500 iterations

of consuming the points $P_{\text {consume }}$ to get the balance again.

We mentioned previously that the action of paying points can be associated with an alternative action to answering that could lead to an answer to the request. For example, the alternative action can be recommending another user to answer the request. In this case, the system will try to notify the recommended user using emails, SMSs or push notifications depending on the implementation of the system. This will raise the probability of the question to be answered. However, too many of these notifications could be considered as a spam to some users. Nevertheless, any other alternative action to answering set by the system designer can be used, and it would be a big plus if it helped in providing an answer.

\section{RELATED WORK}

In line with our study, some studies analysed the problem of motivating users, selfish and cooperative, in the context of online communities in general [1]. In these studies, most of the attempts to analyse the problem are experimental with many uncontrolled variables in established online communities. There are few attempts in using computer simulations to predict the effects of different incentive mechanisms in online communities [8] [9]. Each focused on the results of applying specific incentive mechanism techniques according to economics, behavioural economics and psychology theories. [8] studies the user motivation and incentive mechanisms for participants of an online community for sharing articles and URLs. [9] simulates the effects of different kinds of moderation on the participation of the participants of an online community using an agent based model.

In our model, we use the approach of game theory and more specifically, the prisoner's dilemma game and tit-for-tat strategies, to simulate the behaviour of the participants and analyse it.

In a previous research we have shown the classical Tit for Tat strategy where the player looks back at the previous move by the other player and acts upon it. Such a strategy will affect the survivability of the group negatively where the users will stop collaborating with each other as a result of the negative histories between them. While another strategy, the generous Tit for Tat, has a more relaxed criteria where a player will cooperate with another player generously in a regular basis. Although this strategy solves the problem associated with the previously mentioned classical Tit for Tat strategy, it leads to enticing the free riding behaviour and does not maximise the benefits of the cooperative users [7].

It is worth noting that game theory was used extensively to study the problem of selfish users in the fields of wireless ad-hoc networks, virtual private networks and peer-to-peer networks as well [10], [11], [12].

\section{CONClusion}

In this paper, we proposed a collaborative group in a social application using repeated game theory to simulate the users behaviors. We used the reputation based Tit for Tat strategy to demotivate the free riding behaviour. We concluded that the usage of the reputation-based Tit for Tat strategy while it is good for the group in general, it might be injustice to a number of cooperative users by considering them non cooperative. We proposed giving another chance to the cooperative users to prove that they are not free riding using a points system. As shown in the results, using the points system decreased the number of faults and increased the gains of the cooperative users.

\section{REFERENCES}

[1] J. Vassileva, "Motivating participation in social computing applications: a user modeling perspective," User Modeling and User-Adapted Interaction, pp. 1-25, 2012.

[2] G. Hardin, "The tragedy of the commons," New York, 1968.

[3] P. Kollock, "The economies of online cooperation: Gifts and public goods in cyberspace," Communities in Cyberspace, pp. 220-239, 1999.

[4] R. Boyd and P. J. Richerson, "Punishment allows the evolution of cooperation (or anything else) in sizable groups," Ethology and sociobiology, vol. 13, no. 3, pp. 171-195, 1992.

[5] P. Sloep, "Fostering sociability in learning networks through ad-hoc transient communities," Computer-Mediated Social Networking, pp. 62$75,2009$.

[6] R. Axelrod and W. Hamilton, "The evolution of cooperation," Science, vol. 211, no. 4489, p. 1390, 1981.

[7] A. Al-Dhanhani, R. Mizouni, H. Otrok, and A. Al-Rubai, "Game theoretical analysis of collaborative social applications," in Collaborative Computing: Networking, Applications and Worksharing (CollaborateCom), 2012 8th International Conference on, pp. 628-634, IEEE, 2012.

[8] Y. Mao, J. Vassileva, and W. Grassmann, "A system dynamics approach to study virtual communities," in System Sciences, 2007. HICSS 2007. 40th Annual Hawaii International Conference on, pp. 178a-178a, IEEE, 2007.

[9] Y. Ren and R. Kraut, "A simulation for designing online community: Member motivation, contribution, and discussion moderation," Information Systems Research, 2011.

[10] V. Srivastava, J. Neel, A. MacKenzie, R. Menon, L. DaSilva, J. Hicks, J. Reed, and R. Gilles, "Using game theory to analyze wireless ad hoc networks," Communications Surveys \& Tutorials, IEEE, vol. 7, no. 4, pp. 46-56, 2005.

[11] J. Robert, H. Otrok, A. Quttoum, and R. Boukhris, "A distributed resource management model for virtual private networks: Tit-for-tat strategies," Computer Networks, 2011.

[12] D. Peng, W. Liu, C. Lin, Z. Chen, and X. Peng, "Enhancing tit-for-tat strategy to cope with free-riding in unreliable p2p networks," in Internet and Web Applications and Services, 2008. ICIW'08. Third International Conference on, pp. 336-341, IEEE, 2008.

\section{Creative Commons Attribution License 4.0 (Attribution 4.0 International, CC BY 4.0)}

This article is published under the terms of the Creative Commons Attribution License 4.0 https://creativecommons.org/licenses/by/4.0/deed.en_US 